\title{
Validez, confiabilidad y percentiles de un cuestionario que mide los niveles de actividad en adolescentes que viven a moderada altitud del Perú \\ Validity, reliability, and percentiles of a questionnaire measuring activity levels in adolescents living at moderate altitude in Peru \\ *Marco Antonio Cossio-Bolaños, **Ruben Vidal-Espinoza, ***Jose Sulla-Torres, ****Cristian Luarte-Rocha, *****Jaime Pacheco-Carrillo, *Rossana Gomez-Campos \\ *Universidad Católica del Maule (Chile), **Universidad Católica Silva-Henriquez, Santiago (Chile), ***Universidad Nacional de San Agustín de Arequipa (Perú), ****Universidad San Sebastián (Chile), *****Universidad del Bio Bio (Chile)
}

Resumen. La evaluación y monitorización del nivel de actividad física (AF) debe ser realizada constantemente. Los objetivos del estudio fueron a) Verificar la validez y confiabilidad de un cuestionario que mide el nivel de AF y b) desarrollar percentiles para clasificar los niveles de AF de escolares según edad y sexo. Se efectuó un estudio de descriptivo de corte transversal en 6 establecimientos Públicos de la Provincia de Arequipa, Perú. Fueron encuestados 1,339 adolescentes de ambos sexos (661 hombres y 678 mujeres). Se utilizó un cuestionario de 11 preguntas para medir la AF. Se validó por medio de análisis factorial confirmatorio y la fiabilidad por medio de consistencia interna. Se desarrollaron percentiles. La edad promedio de los hombres fue de 15,24 $\pm 1,82$ años y de las mujeres de $15,39 \pm 1,64$ años. Las saturaciones oscilaron entre 0.30 a 0.55. El modelo de la medida de adecuación de KMO fue de $0.83\left(X^{2}=2206.1\right.$, gl=55, p<0,000). El \% de explicación de la varianza para el tipo de AF fue de 30.0\%, para frecuencia AF 10.7\%, duración AF 9.0\% e intensidad AF 7.9\%. El Alpha de Cronbach fue de $\mathrm{r}=0.71$. Se propusieron percentiles para cada rango de edad y sexo. Los puntos de corte propuestos fueron: Bajo ( $<$ p15), moderado (p15-p85) y alto nivel de AF ( $>$ p85). Se ha verificado que el cuestionario de AF utilizado es válido y confiable, además los percentiles desarrollados son una herramienta útil para diagnosticar, clasificar y monitorizar los niveles de AF de adolescentes que viven a moderar altitud del Perú.

Palabras Clave: Cuestionario; Actividad física; Validez; Confiabilidad, adolescentes.

Abstract. The evaluation and monitoring of physical activity (PA) levels must be carried out constantly. The objectives of the study were a) To verify the validity and reliability of a questionnaire that measures the level of PA and b) to establish percentiles to classify PA levels of schoolchildren by age and gender. A descriptive cross-sectional study was carried out in 6 public establishments in the Province of Arequipa, Peru. A total of 1,339 adolescents (661 boys and 678 girls) were surveyed. A questionnaire comprising 11 items was used to measure PA. Validation was carried out using confirmatory factor analysis, whereas reliability was assessed by means of internal consistency. Percentiles were established. The average age of boys was $15.24 \pm 1.82$ years old, whilst girls were $15.39 \pm 1.64$ years old. Saturations ranged from 0.30 to 0.55 . KMO adequacy measure was $0.83(X 2=2206.1, \mathrm{df}=55, \mathrm{p}<0.000)$. The variance explanation \% for the type of PA was 30.0\%, for PA frequency 10.7\%, for PA duration 9.0\%, and for PA intensity 7.9\%. Cronbach's Alpha was $r=0.71$. Percentiles were proposed for each age and gender range. The proposed cut points were: Low ( $<\mathrm{p} 15)$, moderate (p15-p85) and high level of PA (> p85). The used PA questionnaire is valid and reliable; in addition, the developed percentiles are a useful tool to diagnose, classify, and monitor PA levels of adolescents who live at moderate altitudes in Peru.

Keywords: Questionnaire; Physical activity; Validity; Reliability, adolescents.

\section{Introducción}

La actividad física (AF) regular es un importante componente en la lucha contra la obesidad y condiciones crónicas de salud (WHO, 2009). A pesar de tener beneficios psicosociales y sanitarios, los niveles de AF están disminuyendo significativamente en niños, adolescentes, jóvenes y adultos de diversas partes del mundo (Nader, Bradley, Houts \& McRitchie, 2008; Ortega, 2010; Yañez-Silva, Hespanhol, Gómez-Campos \& Cossio-Bolaños, 2014; DankulincovaVeselska, Sigmundova \& Madarasova-Geckova, 2015).

Las causas y efectos de la inactividad física están bien documentadas, destacando el uso excesivo de las nuevas tecnologías (por ejemplo, avances en el transporte, mejora de la tecnología del transporte), mientras que otros son más sutiles y complejos (por ejemplo, televisores, computadoras, entretenimiento electrónico, internet y conexión inalámbrica de dispositivos de comunicación) (Hallal et al., 2012).

Debido a estas razones, las sociedades contemporáneas

Fecha recepción: 13-12-18. Fecha de aceptación: 08-05-19 Rossana Gomez-Campos

rossaunicamp@gmail.com actualmente están cambiando rápidamente los patrones de AF y están adquiriendo comportamientos sedentarios. En ese sentido, el Perú no está ajeno a esta realidad, puesto que hay evidencias científicas que reflejan bajos niveles de AF en adolescentes y adultos (Mamani, Alvarez-Dong \& Espinoza, 2017), así como incrementos significativos de adiposidad corporal en niños y adolescentes (Cossio-Bolaños, Arruda, Andruske, Luarte-Rocha \& Gómez-Campos, 2016).

En general, proporcionar información sobre los niveles de AF en el ámbito escolar es relevante, pues la práctica constante de AF es la piedra angular para el desarrollo físico de los niños, adolescentes y adultos (Ward, Saunders \& Pate, 2007). De hecho, los niveles de AF deben ser medidos constantemente, no sólo en adultos, sino también en niños y adolescentes.

Por lo tanto, para valorar la AF no sólo es necesario de técnicas e instrumentos cualitativos y/o cuantitativos, sino también es necesario disponer de baremos para identificar, clasificar y/o monitorizar los patrones de AF de forma rápida y sencilla.

En ese contexto, ante la necesidad de contar con un instrumento local y actualizado que permita la autoadministración a grandes poblaciones de forma válida y 
confiable, y además cuente con percentiles para clasificar los niveles de AF, este estudio se propuso como objetivos: a) verificar la validez y confiablidad de un cuestionario que mide el nivel de actividad física y b) desarrollar percentiles para clasificar los niveles de AF de escolares según edad y sexo. Esta información es relevante para el sistema escolar regional y nacional, puesto que disponer de un instrumento válido y confiable para medir los patones de AF podría originar nuevas líneas de investigación de interés social (Infante, Rey-Baltar, Echeazarra, 2019) y geográfico, especialmente cuando se trata de estudiar escolares que viven a moderada altitud del Perú.

\section{Metodología}

\section{Tipo de estudio y muestra}

Se efectuó un estudio de tipo descriptivo de corte transversal en 6 establecimientos Públicos de la Provincia de Arequipa, Perú. Esta ciudad está ubicada a 2320msnm. El rango de edad de los adolescentes oscila entre los 11,0 a 18,9 años. La edad promedio de los hombres fue de 15,24 $\pm 1,82$ años y de las mujeres de 15,39 1 1,64 años. Por lo general, en este país, los escolares que asisten a escuelas públicas pertenecen a la condición socioeconómica media.

Fueron encuestados 1,339 adolescentes de ambos sexos (661 hombres y 678 mujeres) IC: 95\%. Los escolares fueron seleccionados de forma probabilística (estratificada). El número de elementos muestrales de cada estrato fue directamente proporcional al tamaño del estrato dentro de la población (11,800 alumnos, 5880 hombres y 5920 mujeres), considerando la edad y sexo. Por ejemplo, los estratos incluyen rangos de edad desde 11,0 a 11,9, 12,0 a 12,9 hasta completar los 18,9 años.

Se incluyeron en el estudio a los adolescentes, cuyos padres firmaron el consentimiento informado y los que firmaron el asentimiento. Se excluyeron a los que se encontraban fuera de rango de edad y a los que no completaron el cuestionario. El estudio pertenece a un proyecto de investigación desarrollado en la Universidad Nacional de San Agustín, el cual, se efectuó de acuerdo a la declaración de Helsinki y al comité de ética local (UNSA).

\section{Técnicas y Procedimientos}

Para medir el nivel de actividad física se utilizó la técnica de la Encuesta. El instrumento utilizado fue el cuestionario propuesto por Gómez-Campos, Vilcazán, Arruda, Hespañol \& Cossio-Bolaños (2012) (Anexo 1). El instrumento está estructurado en 4 dimensiones: Tipo, duración, frecuencia e intensidad. Mide los patrones de AF durante la última semana. Todo el instrumento tiene 11 preguntas y fue aplicado de forma tradicional a lápiz y papel. Este instrumento fue propuesto hace más de 8 años, por lo que una nueva validación permitiría su actualización. Además, se elaboró una hoja de cálculo para determinar los patrones de AF por edad y sexo (Anexo1). Este procedimiento permite la entrega de resultados en tiempo real. El procedimiento estuvo a cargo de 4 evaluadores con experiencia en técnicas de encuesta. La recolección de información se efectúo durante los meses de abril a julio del 2017. Los cuestionarios fueron aplicados en horarios de clases de Educación Física (8:00 - 14:30hrs).
Cada encuestado tuvo un lapso de 10 a 15 minutos para poder responder las preguntas del instrumento.

La edad decimal se determinó registrando el día, mes y año de nacimiento, así como la fecha de evaluación.

\section{Validez, confiabilidad y baremos}

La validación del instrumento se efectuó por medio de la técnica de análisis factorial confirmatoria. La confiabilidad se determinó por medio de consistencia interna. La propuesta de baremación se efectúo por medio de percentiles para cada sexo y edad. El p $<15$ significa bajo nivel de AF, e»p15 a p85 moderado nivel de AF, y e»p85 indica elevado nivel de AF (Cossio-Bolaños, Méndez Cornejo, Luarte-Rocha, Vargas-Vitoria, Canqui-Flores \& Gomez-Campos, 2017).

\section{Análisis estadístico}

Se verificó la normalidad de los datos por medio de Kolmoronov-Smirnov. Se calculó la estadística descriptiva de media aritmética $(\mathrm{X})$, desviación estándar (DE), frecuencias, porcentajes y rango. Los percentiles se calcularon por medio del método LMS (Cole, Bellizzi Flegal \& Dietz, 2000). Para ello se aplicó el procedimiento de máxima probabilidad de penalización a efecto de crear tres curvas: L(t) Box-Cox Power, M(t) mediana y S(t) Coeficiente de variación. Se consideró los percentiles (p15, p50, p85). La comparación entre ambos sexos se efectuó por medio de test de student para muestras independientes. Para la validez del instrumento se utilizó el método multivariado de análisis factorial confirmatorio con la técnica de extracción de componentes principales, seguida de rotación Varimax y Kaiser-Meier-Olkin (KMO).

Adicionalmente, se llevó a cabo un análisis con el Software EQS 6.2 para confirmar que, efectivamente, ajustaba bien para cuatro factores. Como indicador de bondad de ajuste se utilizó el índice de ajuste comparativo de Bentler (CFI, comparative fit index), así como el índice chi-cuadrado divido entre los grados de libertad $(\div 2 / \mathrm{gl})$ y la raíz cuadrada de la media residual estandarizada (SRMS, Standardized Root Mean Square Residual, Error cuadrático medio de aproximación, RMSEA). Para que existiera un buen ajuste, se debería obtener un CFI cercano a 0,9 o superior, un valor de $\div 2 / \mathrm{gl}$ menor que 4 y un RMSEA menor de 0,08 (Hu \& Bentler, 1999).

Para generar las curvas por edad y sexo, los datos previamente fueron normalizados, luego se realizó una transformación de potencia de Box-Cox. La fiabilidad se determinó por medio del alpha de Cronbach. En todos los casos se consideró un nivel de significancia de 95\% ( $<<0,05)$. Los resultados fueron procesados en planillas de Excel y SPSS 18.0.

\section{Resultados}

Las dimensiones por edad y sexo que caracterizan a la muestra estudiada se observan en la tabla 1. En el tipo y duración de la AF, los hombres mostraron mayores puntajes a los 11 años y desde los 15 hasta los 18 años en relación a las mujeres $(\mathrm{p}<0,05)$. No se observó diferencias significativas en la frecuencia e intensidad en todos los rangos de edad ( $p>0,05)$. En general, en la AF total, los hombres reflejaron valores medios más altos respecto a las mujeres $(\mathrm{p}<0,05)$. 
Tabla 1.

Características de la muestra estudiada

\begin{tabular}{|c|c|c|c|c|c|c|c|c|c|c|c|}
\hline \multirow{2}{*}{ Edades } & \multicolumn{3}{|c|}{ Tipo AF } & \multicolumn{2}{|c|}{ Frecuencia AF } & \multicolumn{2}{|c|}{ Duración AF } & \multicolumn{2}{|c|}{ Intensidad AF } & \multicolumn{2}{|c|}{$\mathrm{AF}$ (total) } \\
\hline & $\mathrm{n}$ & $\mathrm{X}$ & $\mathrm{DE}$ & $\mathrm{X}$ & $\mathrm{DE}$ & $\mathrm{X}$ & $\mathrm{DE}$ & $\mathrm{X}$ & $\mathrm{DE}$ & $\mathrm{X}$ & $\mathrm{DE}$ \\
\hline \multicolumn{12}{|l|}{ Hombres } \\
\hline 11,0-11,9 & 28 & $10,8^{*}$ & 2,9 & 5,7 & 1,3 & $9,1^{*}$ & 8,0 & 6,6 & 1,4 & $32,3^{*}$ & 9,1 \\
\hline $12,0-12,9$ & 86 & 10,3 & 2,9 & 5,3 & 1,4 & 6,9 & 2,6 & 6,2 & 1,7 & $28,7 *$ & 6,3 \\
\hline $13,0-13,9$ & 40 & 9,7 & 3,1 & 5,4 & 1,7 & 7,6 & 2,7 & 7,0 & 1,8 & $29,7 *$ & 6,8 \\
\hline $14,0-14,9$ & 97 & 9,7 & 2,6 & 5,2 & 1,4 & 7,4 & 4,7 & 6,6 & 1,8 & $29,0 *$ & 7,2 \\
\hline $15,0-15,9$ & 135 & $9,8^{*}$ & 2,9 & 5,4 & 1,5 & $7,7^{*}$ & 2,6 & 7,0 & 1,6 & $29,9 *$ & 6,7 \\
\hline $16,0-16,9$ & 129 & $9,9 *$ & 3,0 & 5,2 & 1,6 & $7,8^{*}$ & 2,8 & 6,9 & 1,8 & $29,9 *$ & 7,2 \\
\hline $17,0-17,9$ & 82 & $9,6^{*}$ & 3,1 & 5,2 & 1,6 & $7,5^{*}$ & 3,0 & 6,8 & 1,9 & $29,1^{*}$ & 7,8 \\
\hline $18,0-18,9$ & 64 & $10,0^{*}$ & 2,9 & 5,1 & 1,6 & $7,5^{*}$ & 2,7 & 6,8 & 1,5 & $29,4^{*}$ & 6,8 \\
\hline Total & 661 & $9,9^{*}$ & 2,9 & 5,3 & & $7,6^{*}$ & 3,5 & & 1,7 & $29,5^{*}$ & 7,1 \\
\hline \multicolumn{12}{|l|}{ Mujeres } \\
\hline $11,0-11,9$ & 22 & 8,8 & 2,4 & 6,9 & 1,6 & 6,8 & 2,8 & 6,1 & 1,4 & 28,6 & 9,6 \\
\hline 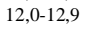 & 69 & 9,3 & 2,8 & - & 1,3 & 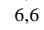 & 2,6 & & 1,8 & 27,2 & 6,6 \\
\hline $13,0-13,9$ & 26 & 9,8 & 3,0 & 5,4 & 1,6 & 7,2 & 2,7 & 6,2 & 1,6 & 28,7 & 6,3 \\
\hline $14,0-14,9$ & 88 & 8,3 & 2,7 & 5 & 1,5 & 6,3 & 2,7 & 6 & 1,6 & 26,1 & 6,1 \\
\hline $15,0-15,9$ & 173 & 8,2 & 4,2 & 4,8 & 1,4 & 6,4 & 2,8 & 6,2 & 1,6 & 25,7 & 7,0 \\
\hline $16,0-16,9$ & 166 & 8,1 & 2,6 & 4,6 & 1,4 & 6,1 & 2,8 & 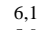 & 1,5 & 24,9 & 6,4 \\
\hline $17,0-17,9$ & 82 & 8,1 & 2,2 & 4,5 & 1,3 & 6,0 & 2,3 & 5,9 & 1,3 & 24,6 & 4,9 \\
\hline $18,0-18,9$ & 52 & 7,9 & 2,6 & 4,2 & 1,2 & 5,9 & 2,5 & 6, & 1,7 & 24,2 & 6,2 \\
\hline Total & 678 & 8,3 & 3,1 & 4,8 & 2,1 & 6,3 & 2,7 & 6,2 & 1,5 & 25,7 & 6,6 \\
\hline
\end{tabular}

significativa $(\mathrm{p}<0.05)$

Los 11 ítems fueron validados por medio de análisis factorial confirmatorio. Las saturaciones (comunalidades) oscilaron entre 0.30 a 0.55 , además el modelo la medida de adecuación de KMO mostró un valor de 0.83 (X2= 2206.1, $\mathrm{gl}=55, \mathrm{p}<0.000)$. El \% de explicación de la varianza para el tipo de $\mathrm{AF}$ fue de $30.0 \%$, para frecuencia AF $10.7 \%$, duración $\mathrm{AF}$ 9.0\% e intensidad AF 7.9\%. El índice de ajuste comparativo de Bentler (CFI) fue de 0,896 , un $\div 2$ /gl de 3,995 y un RMSEA de 0,037 (con un intervalo al 90\% de confianza de 0,0231 y 0,0366 ) que expresa un buen ajuste. Por lo tanto, se confirma que el modelo factorial de 4 factores presenta un buen ajuste. Los valores de confiabilidad oscilan entre $r=0.69-0.75$, y el instrumento en general mostró un alpha de 0.71. La figura 1 , muestra los resultados obtenidos.

Los percentiles construidos por medio del método LMS se pueden observar en la tabla 2. Se consideró los puntos de corte de: p15, p50 y p85. Estos percentiles sirven para clasificar los niveles de AF por edad y sexo.

\section{Discusión}

La actividad física puede ser estimada objetivamente y/o subjetivamente, sin embargo, debido a su viabilidad y costo, las medidas subjetivas como los cuestionarios actualmente se están convirtiendo en una herramienta de uso cotidiano en los colegios.

De hecho, este estudio, tuvo como objetivo inicial verificar la validez y confiabilidad de un cuestionario de AF en

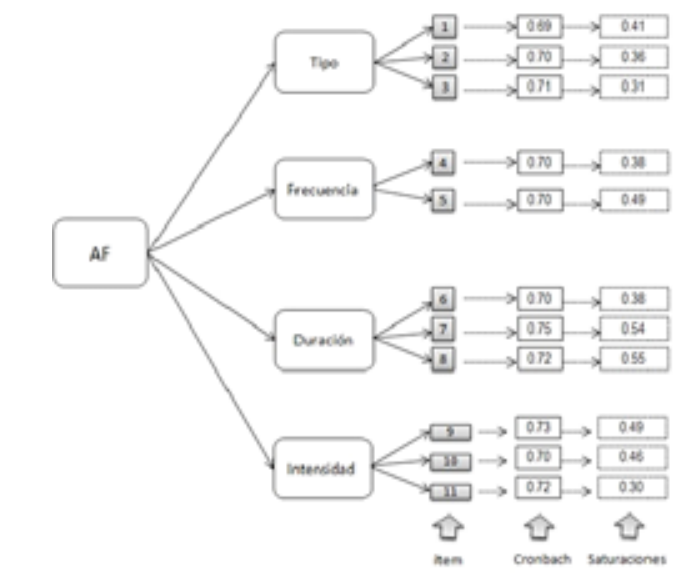

Figura 1. Valores de validez (análisis factorial confirmatorio) y confiabilidad (consistenci interna) del cuestionario de AF en adolescentes de ambos sexos. adolescentes. Estos hallazgos han demostrado que tras la validación por constructo (análisis confirmatorio), este instrumento evidenció saturaciones (comunalidades) superiores a 0,30 y las medidas de adecuación $(\mathrm{KMO}=0.85)$ fueron similares a otros estudios utilizando el mismo instrumento $(4,10,11)$ y otros cuestionarios con similares temáticas (Fernandez-Cabrera, Medina-Anzano, Herrera-Sanchez, Rueda-Mendez \& Fernandez, 2011; Cossio-Bolanos, Vasquez, Luarte-Rocha, Sulla-Torres \& Gomez Campos, 2016), e inclusive él \% de explicación de la varianza del instrumento en su totalidad fue de $57,7 \%$.

Además en relación a la confiabilidad, el instrumento utilizado reflejó valores confiables (á=0,71) al igual que varios estudios que utilizaron el mismo instrumento $(4,11)$ y otros con similares características (Cossio-Bolanos, Vasquez, Luarte-Rocha, Sulla-Torres \& Gomez Campos, 2016; LimaSerrano, Lima-Rodriguez, Saez-Bueno, 2012).

En esencia, estos resultados, evidentemente permitieron contrastar las teorías sustantivas de la escala utilizada en este estudio. Esto debido al cumplimiento de los requisitos mínimos que Caspersen, Nixon \& Durant (1998) destacan para los cuestionarios de AF. Por ejemplo, el instrumento utilizado, no altera el comportamiento de la población, ya que inicialmente fue desarrollado y propuesto para adolescentes del Perú que viven a moderada altitud, además en términos de adaptación es práctico y de fácil aplicación para adolescentes. En términos de control de calidad, el presente instrumento cumple con los requisitos de validez y confiabilidad, independientemente del tipo y/o técnica utilizada.

\begin{tabular}{|c|c|c|c|c|c|c|c|c|c|c|c|c|}
\hline \multirow[b]{2}{*}{ Edades } & \multicolumn{6}{|c|}{ Hombres } & \multicolumn{6}{|c|}{ Mujeres } \\
\hline & $\mathrm{L}$ & M & S & P15 & P50 & P85 & $\mathrm{L}$ & M & $\mathrm{s}$ & P15 & P50 & P85 \\
\hline & \multicolumn{12}{|c|}{ Tipo AF } \\
\hline $11,0-11,9$ & 1,70 & 10,90 & 0,25 & 8 & 11 & 14 & 1,38 & 8,99 & 0,28 & 6 & 9 & 12 \\
\hline $12,0-12,9$ & 1,48 & 10,44 & 0,26 & 7 & 10 & 13 & 1,16 & 9,22 & 0,32 & 6 & 9 & 12 \\
\hline $13,0-13,9$ & 1,28 & 10,02 & 0,27 & 7 & 10 & 13 & 0,95 & 9,03 & 0,35 & 6 & 9 & 12 \\
\hline $14,0-14,9$ & 1,16 & 9,81 & 0,28 & 7 & 10 & 13 & 0,75 & 8,38 & 0,37 & 5 & 8 & 12 \\
\hline $15,0-15,9$ & 1,14 & 9,88 & 0,29 & 7 & 10 & 13 & 0,60 & 7,90 & 0,38 & 5 & 8 & , \\
\hline $16,0-16,9$ & 1,19 & 9,96 & 0,29 & 7 & 10 & 13 & 0,52 & 7,86 & 0,36 & 5 & 8 & 11 \\
\hline $17,0-17,9$ & 1,25 & 9,91 & 0,29 & 7 & 10 & 13 & 0,54 & 7,89 & 0,34 & 5 & 8 & 11 \\
\hline \multirow[t]{2}{*}{$18,0-18,9$} & 1,29 & 9,95 & 0,30 & 7 & 10 & 13 & 0,62 & 7,83 & 0,32 & 5 & 8 & 11 \\
\hline & \multicolumn{12}{|c|}{ Frecuencia AF } \\
\hline 11,0-11,9 & 1,08 & 5,58 & 0,25 & 4 & 6 & 7 & $\begin{array}{l}-0,87 \\
\end{array}$ & 5,17 & 0,29 & 4 & 5 & \\
\hline $12,0-12,9$ & 0,98 & 5,39 & 0,26 & 4 & 5 & 7 & 0,20 & 5,05 & 29 & 4 & 5 & 7 \\
\hline $13,0-13,9$ & 0,91 & 5,30 & 0,28 & 4 & 5 & 7 & 0,69 & 5,13 & 29 & 4 & 5 & r \\
\hline $14,0-14,9$ & 0,88 & 5,25 & 0,28 & 4 & 5 & 7 & 0,62 & 4,95 & 29 & 4 & 5 & 7 \\
\hline $15,0-15,9$ & 0,89 & 5,32 & 0,28 & 4 & 5 & 7 & 0,38 & 4,70 & 29 & 3 & 5 & 6 \\
\hline $16,0-16,9$ & 0,93 & 5,28 & 0,28 & 4 & 5 & 7 & 0,50 & 4,56 & 0,29 & 3 & 5 & 6 \\
\hline $17,0-17,9$ & 0,93 & 5,24 & 0,28 & 4 & 5 & 7 & 0,49 & 4,41 & 29 & 3 & 4 & c \\
\hline \multirow{2}{*}{$18,0-18,9$} & 0,93 & 5,15 & 0,28 & 4 & 5 & 7 & 0,21 & 4,10 & 0,28 & 3 & 4 & 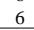 \\
\hline & \multicolumn{12}{|c|}{ Duración AF } \\
\hline $11,0-11,9$ & 0,52 & 7,17 & 0,38 & 5 & 7 & 10 & 0,82 & 70 & 39 & 4 & 7 & 10 \\
\hline $12,0-12,9$ & 0,33 & 6,76 & 0,39 & 4 & 7 & 10 & 0,73 & 62 & 40 & 4 & 7 & 10 \\
\hline $13,0-13,9$ & 0,17 & 6,78 & 0,40 & 4 & 7 & 10 & & 52 & & 4 & 7 & 10 \\
\hline $14,0-14,9$ & 0,13 & 6,95 & 0,40 & 5 & 7 & 10 & 0,53 & 6,29 & 0,42 & 4 & 6 & 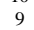 \\
\hline $15,0-15,9$ & 0,30 & 7,27 & 0,39 & 5 & 7 & 10 & 0,41 & 16 & 42 & 4 & 6 & 9 \\
\hline $16,0-16,9$ & 0,55 & 7,54 & 0,38 & 5 & 8 & 11 & 0,34 & 5,96 & 42 & 4 & 6 & 9 \\
\hline $17,0-17,9$ & 0,80 & 7,57 & 0,37 & 5 & 8 & 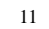 & 030 & 73 & 12 & 4 & 6 & \\
\hline \multirow[t]{2}{*}{$18,0-18,9$} & 1,04 & 7,50 & 0,36 & 5 & 8 & 10 & 0,26 & 5,52 & 0,41 & 4 & 6 & 8 \\
\hline & \multicolumn{12}{|c|}{ Intensidad AF } \\
\hline 11,0-11,9 & 0,92 & 6,34 & 0,26 & 5 & 6 & 8 & 0,76 & 91 & 28 & 4 & 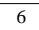 & 0 \\
\hline $12,0-12,9$ & 0,76 & 6,37 & 0,26 & 5 & 6 & 8 & 0,62 & 11 & 27 & 5 & 6 & 8 \\
\hline $13,0-13,9$ & 0,60 & 6,57 & 0,26 & 5 & 7 & 8 & 0,50 & 6,22 & 26 & 5 & 6 & 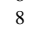 \\
\hline $14,0-14,9$ & 0,50 & 6,69 & 0,25 & 5 & 7 & 9 & 044 & 26 & 5 & 5 & 6 & 8 \\
\hline 15 & 0,43 & 6,83 & 0,24 & 5 & 7 & 9 & 47 & 16 & 24 & 5 & 6 & 8 \\
\hline $16,0-16,9$ & 0,42 & 6,86 & 0,24 & 5 & 7 & 9 & 0,59 & 5,99 & 0,24 & 5 & 6 & 8 \\
\hline $17,0-17,9$ & 0,46 & 6,78 & 0,24 & 5 & 7 & 0 & & 5,93 & 24 & 5 & 6 & 8 \\
\hline \multirow[t]{2}{*}{$18,0-18}$, & 0,49 & 6,72 & 0,23 & 5 & 7 & 8 & 0,80 & 6,10 & 0,25 & 5 & 6 & 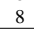 \\
\hline & \multicolumn{12}{|c|}{ Actividad Física (Total) } \\
\hline $11,0-11,9$ & 1,77 & 30,37 & 0,19 & 24 & 30 & 36 & $-0,39$ & 27,17 & 0,30 & 20 & 27 & 38 \\
\hline $12,0-12,9$ & 1,30 & 29,34 & 0,20 & 23 & 29 & 35 & 0,10 & 26,57 & 0,28 & 20 & 27 & 35 \\
\hline $13,0-13,9$ & 0,90 & 29,08 & 0,22 & 23 & 29 & 36 & 0,39 & 26,35 & 0,27 & 20 & 26 & 34 \\
\hline $14,0-14,9$ & 0,74 & 29,16 & 0,23 & 22 & 29 & 36 & 0,48 & 25,79 & 0,26 & 19 & 26 & 33 \\
\hline $15,0-15,9$ & 0,88 & 29,63 & 0,23 & 23 & 30 & 37 & 0,52 & 25,25 & 0,26 & 19 & 25 & 32 \\
\hline $16,0-16,9$ & 1,17 & 29,90 & 0,24 & 22 & 30 & 37 & & 24,68 & 0,25 & 19 & 25 & 31 \\
\hline $17,0-17,9$ & 1,40 & 29,79 & 0,24 & 22 & 30 & 37 & 0,69 & 24,27 & 0,24 & 18 & 24 & 31 \\
\hline $18,0-18,9$ & 1,57 & 29,59 & 0,23 & 22 & 30 & 36 & 0,79 & 24,09 & 0,24 & 18 & 4 & 30 \\
\hline
\end{tabular}


La mayoría de los cuestionarios fueron desarrollados y propuestos en inglés. Estos instrumentos en general necesitan ser adaptados a otros idiomas, los que muchas veces difieren entre poblaciones (Platat \& Jarrar, 2012), por lo que es necesario traducciones, adaptaciones y validaciones acordes a los contextos sociales, culturales y geográficos.

Un segundo objetivo de este estudio, apuntó al desarrollo de valores normativos por medio de percentiles. Esta información sirve para comparar las puntuaciones obtenidas de una persona en particular con los valores normativos de una población general de la misma edad y sexo.

Desde esa perspectiva, los puntos de corte que se adopten, por lo general sirven para determinar los niveles y para identificar las pautas de AF que se deben efectuar según edad y sexo. Los datos normativos pueden ayudar en la interpretación de las puntuaciones de los patrones de AF y proporcionar información individual y colectiva a través del tiempo (Voss, Dean, Gardner, Duncombe \& Harris, 2017).

Los puntos de corte adoptados en esta investigación fueron basados en un estudio anterior (Cossío-Bolaños, Méndez Cornejo, Luarte-Rocha, Vargas-Vitoria, Canqui-Flores \& Gomez-Campos, 2017). Esta herramienta propuesta permitirá contribuir eficientemente al diagnóstico, monitoreo y control de los patrones de AF (Cossio-Bolaños, Arruda, Andruske, Luarte-Rocha \& Gómez-Campos, 2016), además abre la posibilidad de investigar a los escolares que están en riesgo de desarrollar alguna enfermedad crónica y así poder imple-mentar iniciativas dentro del establecimiento o derivarlos a un centro de salud (Moreno, Concha \& Kain). Esto significa que a partir de los percentiles propuestos es posible clasificar y/o diagnosticar a los adolescentes en tres categorías: en el que se define como bajo nivel de $\mathrm{AF}(<\mathrm{p} 15)$, moderado nivel de AF (p15-p85) y alto nivel de AF (>p85).

En general, la AF es un componente clave para mantener y mejorar la salud, incluida la salud física, mental y emocional (Eime, Young, Harvey, Charity \& Payne, 2013), la que debe ser monitorizada constantemente, en especial en los colegios, puesto que por lo general los colegios son los lugares adecuados para la adquisición de hábitos de vida activo y saludable, así como para promocionar la AF y prevención del sedentarismo (Baena, Cerezo \& Fernández, 2010). Todo ello, es posible a través del uso del cuestionario validado en este estudio y del formulario propuesto (Anexo 1) para la la clasificación en una de las tres categorías propuestas.

En consecuencia, la realización de AF desde edades tempranas puede determinar en buena parte la configuración de hábitos de AF saludables y mantener una condición física adecuada a la lo largo de la vida (Gallego et al, 2016). Su control podría ser efectuado por medio del cuestionario validado, además, su evaluación es posible en tiempo real por medio del siguiente link: www.http://www.reidebihu.net/ act_fis_nivel.php o usando el anexo 1. Este instrumento debe ser introducido en la asignatura de educación física, puesto que dicha disciplina presenta una carga lectiva limitada dentro del currículo (Méndez-Alonso et al, 2015), por lo que el instrumento propuesto, podría ser una alternativa para generar sus propios resultados de forma rápida y precisa.

Las principales limitaciones del presente estudio se basan en el tipo de estudio desarrollado (transversal) y en la ausencia de un método objetivo que permita verificar la vali- dez de criterio, sin perjuicio de estas desventajas, futuros estudios deben considerar estos aspectos para evitar posibles sesgos en sus resultados, además es necesario resaltar algunas ventajas de esta investigación, especialmente las que están relacionadas con el tamaño de la muestra y con la facilidad en su uso y aplicación, ya que es factible su generalización a los escolares del Perú con similares características.

En conclusión, se ha verificado que el cuestionario de AF utilizado en este estudio es válido y confiable, además los percentiles desarrollados son una herramienta útil para diagnosticar, clasificar y monitorizar los niveles de AF de adolescentes que viven a moderar altitud del Perú. Los resultados sugieren su uso y aplicación en los centros escolares como un instrumento de evaluación complementario en la educación física.

\section{Agradecimientos}

A la Universidad Nacional de San Agustín de Arequipa, quien ha financiado el proyecto «Propuesta normativa para valorar los niveles de Actividad Física de los escolares de la provincia de Arequipa», con numero de contrato 15-2016UNSA por el financiamiento otorgado para realizar el artículo.

\section{Referencias}

Baena, A. C. M., Cerezo, C. R., \& Fernández, M. D. (2010). Factores que inciden en la promoción de la actividad físico-deportiva en la escuela desde una perspectiva del profesorado. Cuadernos de psicología del deporte, 10(2), 57-78. https://www.redalyc.org/pdf/2270/ 227018000006.pdf

Brindova, D., Dankulincova-Veselska, Z., Sigmundova, D., Madarasova-Geckova, A. (2015). Age and gender differences in prevalence of screen based behavior, physical activity, and health complaints among Slovak school-aged children. Cent Eur J Public Health. Forthcoming. https://www.questia.com/library/journal/ 1P3-3927574091/age-and-gender-differences-inprevalence-of-screen

Caspersen, C.J., Nixon, P., Durant, R. (1998). Physical activity epidemiology applied to children and adolescents. Exerc Sport Sci Rev, 23:341-403. https://www.ncbi.nlm.nih.gov/ pubmed/9696995

Cole TJ, Bellizzi MC, Flegal KM, Dietz WH. Establishing a standard definition for child overweight and obesity worldwide: International survey. BMJ. 2000; 320:1240-3. https://www.ncbi.nlm.nih.gov/pubmed/10797032

Cossio-Bolaños, M., Arruda, M., Andruske, C., Luarte-Rocha, C., Gómez-Campos, R. (2016). Secular trends of physical growth and abdominal adiposity of school children and adolescents living at a moderate altitude in Peru. Am. J. Phys. Anthropol, 1-8. Doi: 10.1002/ajpa.23121 Cossío-Bolaños, M., Méndez Cornejo J, Luarte-Rocha, C., Vargas-Vitoria, R., Canqui-Flores, B., Gomez-Campos, R. (2017). Patrones de actividad física de adolescentes escolares: validez, confiabilidad y propuesta de percentiles para su evaluación. Rev Chil Pediatr, 88(1):45-54. http:// 
dx.doi.org/10.1016/j.rchipe.2016.07.010

Cossio-Bolanos, M.A., Vasquez, P., Luarte-Rocha, C., SullaTorres, J., Gomez-Campos R. Evaluacion de la autopercepcion de la aptitud fisica y propuesta de normativas en adolescentes escolares chilenos: estudio EAPAF. Arch Argent Pediatr. 2016; 114: 319-28. Doi 10.5546/aap.2016.319

Eime RM, Young JA, Harvey JT, Charity MJ, Payne WRA. Systematic review of the psychological and social benefits of participation in sport for children and adolescents: Informing development of a conceptual model of health through sport. Int. J. Behav. Nutr. Phys. Act. 2013; 10, 98. https://doi.org/10.1186/1479-5868-10-98

Fernandez-Cabrera T, Medina-Anzano S, Herrera-Sanchez IM, Rueda-Mendez S, Fernandez A. Construccion y validación de una escala de autoeficacia para la actividad fisica. Rev Esp Salud Pública. 2011; 85:405-17. https:// www.redalyc.org/pdf/170/17019926009.pdf

Gallego FL, Sánchez AJL, Vacas NE, Zagalaz JC. Influencia del género, la edad y el nivel de actividad física en la condición física de alumnos de educación primaria. Revisión Bibliográfica. Retos: nuevas tendencias en educación física, deporte y recreación. 2016; 29: 129-133.

Gómez-Campos R, Vilcazán E, Arruda M, Hespañol J, CossioBolaños MA. Validación de un cuestionario para la valoración de la actividad física en escolares adolescentes. An Fac med. 2012; 73(4):307-13. http://www.scielo.org.pe/ pdf/afm/v73n4/a07v73n4.pdf

Hallal PC, Andersen LB, Bull FC, Guthold R, Haskell W, Ekelund U; Lancet Physical Activity Series Working Group. Global physical activity levels: surveillance progress, pitfalls, and prospects. Lancet. 2012; 380(9838):247-57. Doi: 10.1016/S0140-6736(12)60646-1

$\mathrm{Hu} \mathrm{L}$, Bentler P. Cut off criteria for fit indexes in covariance structure analysis: Conventional criteria versus new alternatives. Structural equation modeling: a multidisciplinary journal. 1999; 6(1): 1-55.

Infante BorinagaG, Rey-Baltar A, Echeazarra Escuero I. Un nuevo cuestionario para evaluar la procrastinación en la actividad físico-deportiva: PAFD. Retos: nuevas tendencias en educación física, deporte y recreación. 2019; 36: 26-31

Lima-Serrano M, Lima-Rodriguez J, Saez-Bueno A. Diseno y validacion de dos escalas para medir la actitud hacia la alimentacion y la actividad fisica durante la adolescencia. Rev Esp Salud Pública. 2012; 86:253-68. http://
scielo.isciii.es/scielo.php?script=sci_arttext\&pid=S113557272012000300005

Mamani T, Alvarez Dong D, Espinoza Oriundo P. Prevalencia y factores asociados a la baja actividad física de la población peruana. Nutr. clín. diet. hosp. 2017; 37(4):108-115. Doi: $10.12873 / 374 t a r q u$

Méndez-Alonso D, Fernández-Río J, Méndez-Giménez A, Prieto-Saborit JA. Análisis de los currículos autonómicos LOMCE de Educación Física en Educación Primaria. Retos. Nuevas tendencias en Educación Física, Deporte y Recreación. 2015;28:15-20. Recuperado de: https:// goo.gl/YcLqiT

Moreno L, Concha F, Kain J. Intensidad de movimiento de escolares durante clases de educación física de colegios municipales: resultados según el profesional que efectúa las clases. Rev Chil Nutr. 2012; 39(4):123-28. http:// dx.doi.org/10.4067/S0717-75182012000400003

Nader PR, Bradley RH, Houts RM, McRitchie SL, O’Brien M. Moderate-to-vigorous physical activity from ages 9 to 15 years. JAMA. 2008; 300(3):295-305. Doi: 10.1001/ jama.300.3.295.

Platat C, Jarrar A. Reliability and validity of a physical activity questionnaire in children. International Journal of Food Sciences and Nutrition. 2012; 63(6): 637-644. https:// p d f s.s e m a n t i c s c h o la r.org / 6 b c $0 /$ afff254d2639f1df9c42d73d54a58db0bed2.pdf

Ortega FB. Cardiovascular fitness modifies the associations between physical activity and abdominal adiposity in children and adolescents: the European Youth Heart Study. BJSM 2010; 44:256-62

Voss C, Dean PH, Gardner RF, Duncombe SL, Harris KC. Validity and reliability of the Physical Activity Questionnaire for Children (PAQ- C) and Adolescents (PAQ-A) in individuals with congenital heart disease. PLoS ONE. 2017; 12(4): e0175806. https://doi.org/10.1371/ journal.pone.0175806

Ward DS, Saunders RP, Pate RR. Physical Activity Interventions in Children and Adolescents; Human Kinetics: Champaign, IL, USA, 2007.

World Health Organization. Interventions on diet and physical activity: what works. Geneva: WHO; 2009

Yáñez-Silva A, Hespanhol JH, Gómez Campos R, CossioBolaños M. Valoración de la actividad física en adolescentes escolares por medio de cuestionario. Rev Chil Nutr 2014; 41(4). http://dx.doi.org/10.4067/S071775182014000400003

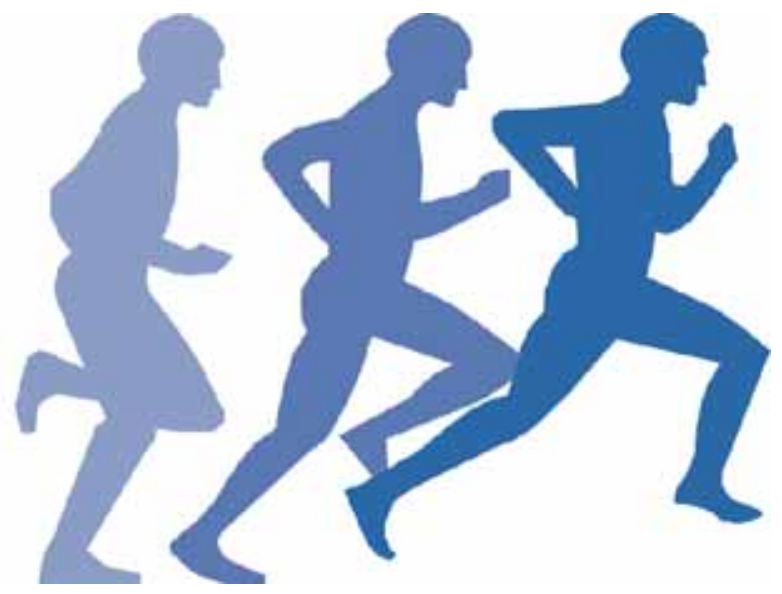

